\title{
Application of blockchain technology to enhance data security of the medical records
}

\author{
B. Ranasinghe ${ }^{1}$, R. Hewapathirana ${ }^{2}$, V.H.W. Dissanayake ${ }^{2}$ \\ 1 Post Graduate Institute of Medicine, University of Colombo, Sri Lanka \\ 2 Faculty of Medicine, University of Colombo, Sri Lanka \\ *buddhikatharindu@gmail.com
}

\begin{abstract}
Introduction: Blockchain is a rapidly evolving data security practice which employs a growing list of records linked using cryptographic hash of the block proceeding, a timestamp and transaction data. Electronic medical records (EMR) are gaining popularity in health sector. Most of these EMRs are based on relational databases. Data tampering is a known issue found in relational data base approach and it is fairly simple to alter a relational data base record with the administrative privileges. This research explores the potentials of blockchain technology with its resistant to modification of the data, as a tamper protection mechanism for medical records.
\end{abstract}

Methods: A blockchain-based program was developed using Proof-of-Work, to maintain the integrity of the digital record independent of access permissions. This was preceded by a series of interviews with clinicians and EMR administers to understand the possible data tampering in association with paper-based and electronic medical records in the State health sector of Sri Lanka. The blockchain algorithm was applied to the Hospital Health Information Management System (HHIMS) clinical database. A security audit was used to assess the tamper-protection capabilities of the relational database.

Results: It was possible to incorporate the blockchain algorithm to the relational database of Hospital HHIMS successfully. An independent blockchain-based data repository was created in association with the native data base of HHIMS. With this approach, a 'block' is being created for each new record added to HHIMS database. Hence, the blockchain generated was instrumental in verifying the authenticity of EMR transactions.

Conclusion: With the increasing penetration of EMR in health sector, the integrity of the digital stored clinical data is becoming a vital necessity. In centralized database administration, possibility of tampering is high. Clinical data can be counted as 'high risk' for such occurrences. The blockchain technology found to be capable of making relational data base tamper-protected by verifying the authenticity of the transactions and data being stored in HHIMS.

Keywords: Blockchain, Relational database, Electronic Medical Record, Tamper protection, HHIMS 